\section{Glukose im Urin}

\author{
K. J. Lackner ${ }^{1}$ und D. Peetz ${ }^{2}$ \\ ${ }^{1}$ Institut für Klinische Chemie und Laboratoriumsmedizin, \\ Universitätsmedizin Mainz, Mainz, Deutschland \\ ${ }^{2}$ Institut für Labormedizin, Helios Klinikum Berlin-Buch, \\ Berlin, Deutschland
}

\section{Synonym(e) Urin-Glukose}

Englischer Begriff glucose in urine

Definition Glukosekonzentration im Urin.

Struktur $>$ Glukose.

Molmasse $>$ Glukose.

Synthese - Verteilung - Abbau - Elimination $>$ Glukose.

Funktion - Pathophysiologie Glukose wird normalerweise sehr effizient tubulär rückresorbiert, sodass beim Gesunden nur wenig Glukose im Urin ausgeschieden wird. Bei Überschreiten der Rückresorptionskapazität bei ca. 160-180 mg/dL Blutglukose kommt es zu einer zunehmenden Glukosurie. Diese sog. Nierenschwelle kann individuell sehr variabel sein.

Untersuchungsmaterial - Entnahmebedingungen Spontanurin.

Probenstabilität Glukose nimmt im Urin ohne entsprechende Zusätze relativ schnell ab, sodass die Bestimmung innerhalb von 2 Stunden erfolgen sollte.

Analytik Uringlukose wird üblicherweise mit trockenchemischen Teststreifenverfahren bestimmt, die auf der Glukoseoxidase-Methode beruhen.

\section{Konventionelle Einheit $\mathrm{mg} / \mathrm{L}$.}

Internationale Einheit $\mathrm{mmol} / \mathrm{L}$.

Umrechnungsfaktor zw. konv. u. int. Einheit 0,00555 .

Referenzbereich - Erwachsene $<0,83 \mathrm{mmol} / \mathrm{L}(<150 \mathrm{mg} / \mathrm{L})$.

Referenzbereich - Kinder S. Erwachsene.

Indikation Wegen der geringen Sensitivität hat die Glukosebestimmung im Urin keinen Platz als Screeningtest in der Primärdiagnostik des Diabetes mellitus. Auch in der Verlaufskontrolle des Diabetikers ist sie wegen der hohen interindividuellen Variabilität der Nierenschwelle und der deshalb schlechten Korrelation zum Blutglukosewert gegenüber der Blutglukosebestimmung als obsolet anzusehen.

Interpretation Erhöhte Glukosewerte im Urin sind nach Ausschluss von Störeinflüssen (peroxidhaltige Reinigungsmittel) immer ein Hinweis auf eine Hyperglykämie und sollten durch entsprechende Diagnostik abgeklärt werden.

Diagnostische Wertigkeit Aufgrund der exzellenten Verfügbarkeit von Methoden zur Blutglukosebestimmung hat die Bestimmung der Glukose im Urin nur noch eine untergeordnete Bedeutung.

\section{Literatur}

Miedema K (2003) Laboratory tests in diagnosis and management of diabetes mellitus. Practical considerations. Clin Chem Lab Med 41:1259-1265 